\title{
Non-classical crystallization of anhydrous Ca and Mg carbonates from the Coorong Lakes, Australia
}

\author{
MAIJA J. RAUdSEPP ${ }^{1 *}$, SiobHAN A.WILSON ${ }^{1}$, BREE \\ MORGAN $^{2}$ AND STEWART FALLON ${ }^{3}$ \\ ${ }^{1}$ Department of Earth and Atmospheric Sciences, University \\ of Alberta, Edmonton, AB, Canada \\ (*correspondence: raudsepp@ualberta.ca) \\ ${ }^{2}$ School of Geosciences, University of Sydney, Camperdown, \\ NSW, Australia \\ ${ }^{3}$ Research School of Earth Sciences, Australian National \\ University, Canberra, ACT, Australia
}

The Coorong Lakes in Australia have long been studied as model environments for low temperature dolomite formation [1]. We took sediment push cores from three Coorong Lakes (Milne Lake, Pellet Lake and North Stromatolite Lake), which collectively cover $\sim 6000$ years of carbonate deposition in these shallow ephemeral lakes. Each lake has a different mineral assemblage of $\mathrm{Ca}$ and $\mathrm{Mg}$ carbonates, which includes aragonite, calcite, very high magnesium calcite (VHMC), Ca-bearing magnesite and/or hydromagnesite.

Our scanning electron microscope (SEM) imaging reveals that VHMC from the Coorong Lakes consists of spherical, sub-micrometre aggregates of nanocrystals, whereas aragonite and magnesite have typical tabular and rhombic habits, respectively. Our high resolution transmission electron microscopy (TEM) analyses and Rietveld refinement results using X-ray diffraction (XRD) patterns show that all anhydrous $\mathrm{Ca}$ and $\mathrm{Mg}$ carbonates are composed of 5-10 nm crystallites. The crystallite size of these carbonate minerals does not change with depth in the lake sediments, suggesting the carbonate nanocrystallites are stable for thousands of years; they neither fuse nor undergo Ostwald ripening. The formation of nanometre-sized crystallites is characteristic of a non-classical pathway, whereby carbonates crystallize from an amorphous precursor to form mesocrystals via oriented attachment [2]. Understanding pathway dependence in the formation of $\mathrm{Ca}$ and $\mathrm{Mg}$ carbonates can provide environmental and geochemical constraints on the precipitation of these minerals. Our work on the Coorong Lakes suggests that non-classical crystallization may be a natural pathway for abiological formation of carbonate sediments.

[1] Rosen et al. (1989) GCA, 53, 661-669. [2] De Yoreo et al. (2015) Science, 349, aaa6760. 